EPJ Web of Conferences 59, 08008 (2013)

DOI: $10.1051 /$ epjconf/20135908008

(C) Owned by the authors, published by EDP Sciences, 2013

\title{
Linear precompensation of FM-to-AM conversion due to frequency conversion system
}

\author{
S. Vidal ${ }^{\mathrm{a}}$, J. Luce and D. Penninckx \\ CEA, DAM, CESTA, BP. 2, 33114 Le Barp, France
}

\begin{abstract}
We report on an experimental demonstration of linear precompensation of nonlinear phase and amplitude transfer functions. We show the effective compensation with a linear all-fiber system of phaseto-amplitude modulation conversion due to a frequency conversion system including plane gratings and a nonlinear crystal.
\end{abstract}

\section{INTRODUCTION}

The Laser MegaJoule (LMJ) in France and the National Ignition Facility (NIF) in the United States are high power laser facilities designed to achieve inertial confinement fusion. Such energetic lasers have to be phase modulated (i.e. frequency modulated) to broaden the optical spectrum in order to suppress stimulated Brillouin scattering and to smooth the speckle pattern illuminating the target. Ideally, pure phase modulations do not affect the temporal shape of the pulse. Nevertheless, propagation through different optical components slightly filters the spectrum and consequently the frequency modulation (FM) is partly converted into amplitude modulations (AMs) [1]. This conversion adversely affects the laser performance and can prevent fusion ignition. Intensity modulations due to linear spectral filters can be entirely and easily compensated with inverse transfer functions. Finally, for the LMJ, amplitude modulations are principally due to non-linear phenomena in the Frequency Conversion System (FCS) and thus the suppression of this FM-to-AM conversion seems to be particularly difficult but of major interest [2].

We report on what we believe to be the first experimental demonstration of the linear precompensation of nonlinear phase and amplitude transfer functions. We show the effective compensation with a linear all-fiber system of AMs due to the FCS.

\section{EXPERIMENTAL SETUP}

We design the following experimental setup in order to exalt the FM-to-AM conversion process and its compensation (see Fig. 1). The laser system is composed of a fiber laser which delivers 3 ns square laser pulses at $\lambda_{0}=1053 \mathrm{~nm}$, an electro-optic phase modulator (EOM) and a regenerative amplifier. A sinusoidal phase modulation $\varphi(t)=m \sin \left(2 \pi f_{m} t\right)$ with $m=8$ and $f_{m}=10 \mathrm{GHz}$ is applied in order to create a $\Delta f=2$. $(m+1) \cdot f_{m}=180 \mathrm{GHz}$ bandwidth spectrum composed of uniformly separated $f_{m}$ spaced Dirac peaks.

At the output of the regenerative amplifier, we obtain a $2 \mathrm{~mm}$ collimated Gaussian beam with an intensity of around $50 \mathrm{MW} / \mathrm{cm}^{2}$. It should be noted that the weak FM-to-AM conversion due to gain

\footnotetext{
ae-mail: sebastien.vidal@cea.fr
}

This is an Open Access article distributed under the terms of the Creative Commons Attribution License 2.0, which permits unrestricted use, distribution, and reproduction in any medium, provided the original work is properly cited. 


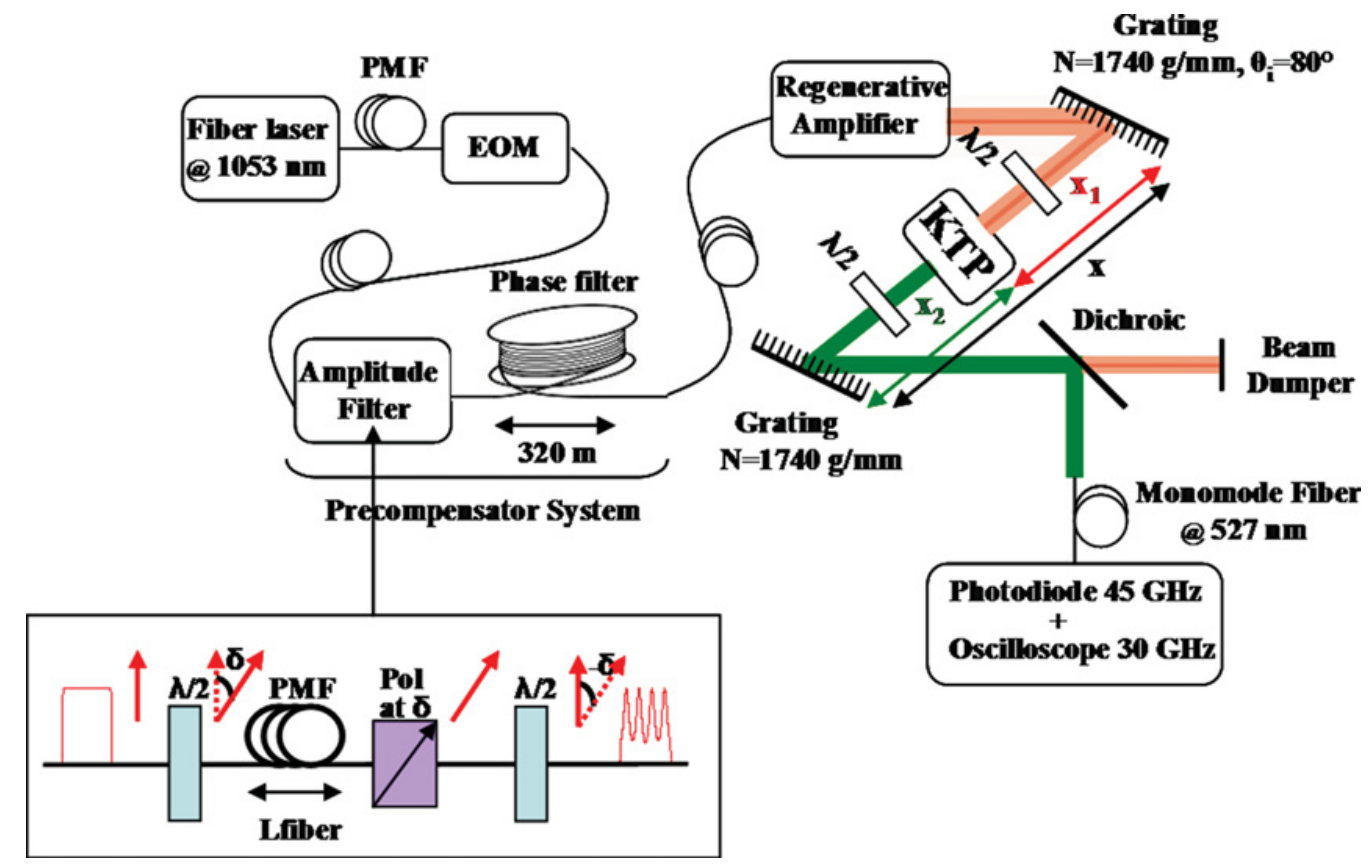

Figure 1. Experimental setup. The amplitude filter is an interferometric filter. The evolution of the polarization is represented by an arrow. (Pol, polarizer; $\lambda / 2$, half-wave plate; PMF, polarization maintaining fiber).

narrowing in the regenerative amplifier is perfectly precompensated. The amplified pulse is then injected into the frequency conversion system composed of two $N=1740 \mathrm{~g} / \mathrm{mm}$ plane gratings and a 5-mmthick KTP (potassium titanyl phosphate) doubler crystal. The distance between the two gold gratings is fixed to $x=46 \mathrm{~cm}$, and the angle of incidence on the first grating is around $\vartheta i \approx 80^{\circ}(\vartheta$ Littrow $\approx 66^{\circ}$ ). This first grating improves the spectral acceptance of the crystal [3] and the second is used to stop the dispersion. A first half-wave plate is used to realize the type-II phase matching condition for the central wavelength and another is inserted before the second grating to optimize the second order of diffraction at $527 \mathrm{~nm}$. We measure the temporal profile of the second harmonic pulse thanks to a monomode fiber at $527 \mathrm{~nm}$ coupled to a $40 \mathrm{GHz}$ bandwidth fibered photodiode and a $30 \mathrm{GHz}$ bandwidth oscilloscope.

We insert the precompensator system (an amplitude filter + a phase filter) in the fibered part of the laser system before amplification for two reasons: it is easier to act on the optical signal when it is less energetic and the regenerative amplifier recovers the energy loss due to the filter. The amplitude filter is composed of a birefringent monomode polarization maintaining fiber (PMF), a half-wave plate to set the state of polarization at an angle $\delta$ of the low axis of the PMF, a fiber polarizer, and a second half-wave plate to set the polarization back to the initial state. The PMF length determines the delay between the two waves propagating in the fiber and the polarizer makes them interfere. We add a Peltier device to precisely adjust the phase recombination $\psi$ between the two waves [4]. As a phase filter, we propose to use the chromatic dispersion of another PMF [5].

\section{RESULTS}

The main results are presented in the Fig. 2. We show that we can consider separately amplitude and phase spectral filters and we demonstrate the performance of an all-fiber system to precompensate a large part of AMs. 

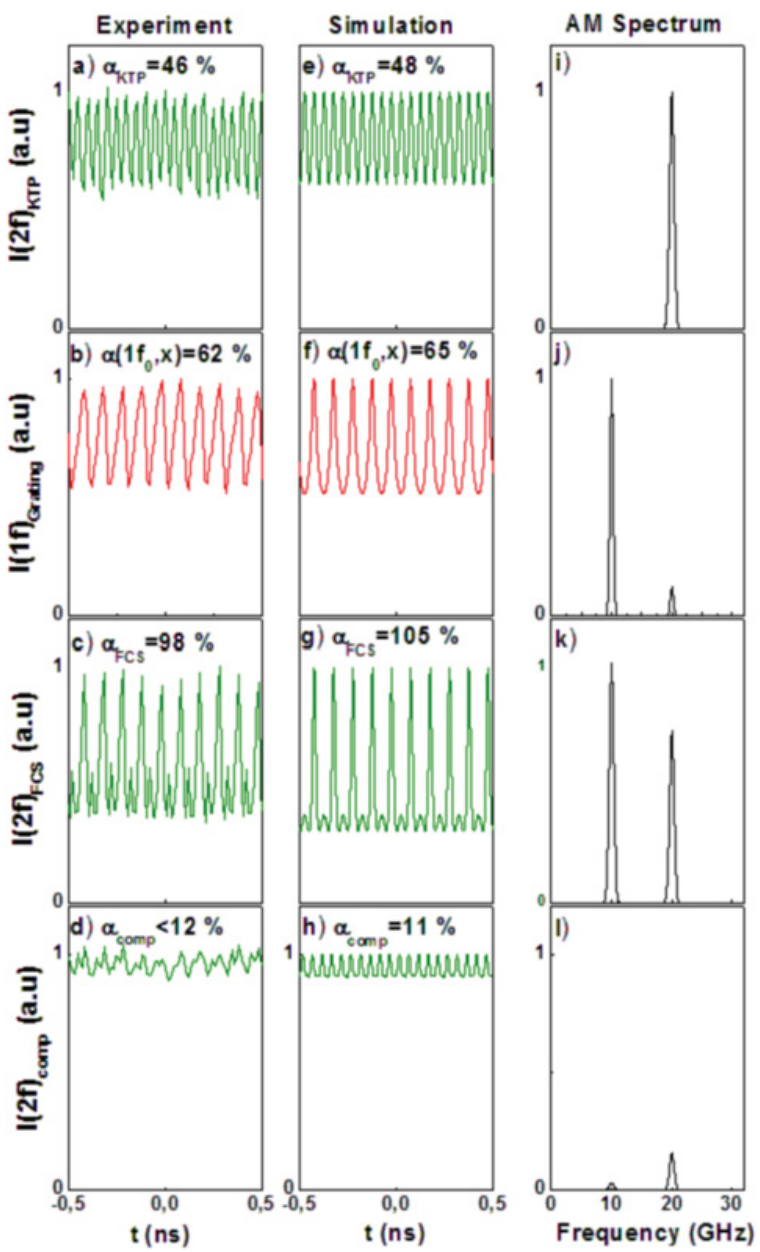

Figure 2. Experimental measurements (zoom on $1 \mathrm{~ns}$ ) of FM-to-AM conversion due to (a) a unique 5-mm-thick KTP crystal, (b) propagation of the fundamental wave between the two gratings, and (c) complete FCS. (d) Compensation of FM-to-AM conversion with the all-fiber system. Figures (e), (f), (g), and (h) correspond to the simulations of (a), (b), (c), and (d), respectively. Figures (i), (j), (k), and (l), respectively, represent the spectrum of AMs for the four measurements.

In the KTP crystal, the main origin of FM-to-AM conversion is the nonlinear amplitude filter of the spectrum due to the group velocity difference between the fundamental and second harmonic waves. We have measured the resulting modulations due to the unique crystal, and we use the distortion criterion $\alpha$ equal to twice the temporal contrast to quantify them [2]. The result is in excellent agreement with those obtained by numerically solving the nonlinear coupled wave equations [cf. Figs. 2(a) and 2(e)]. Concerning the frequency of AMs, we observe only the harmonics of $f_{m}$, mostly $20 \mathrm{GHz}$.

Note that the first plane grating improves the spectral acceptance of the KTP and thus decreases the distortion induced by the crystal [3].

The second origin of FM-to-AM conversion is the propagation after the spatial dispersion induced by the first grating. Figure 2(b) represents an experimental measurement of AMs due to the simple propagation of the fundamental wave between both gratings (without second-harmonic generation). As can be seen in Fig. 2(j), the frequency of AMs induced by this spatial dispersion is mainly $f_{m}$. The propagation can be considered as a linear phase transfer function but when we add the KTP, the 


\section{EPJ Web of Conferences}

FM-to-AM conversion due to the grating becomes nonlinear. Finally, AMs due to our complete FCS are represented in Fig. 2(c).

To precompensate the spectral phase filter, we propose to use the chromatic dispersion of a monomode polarization maintaining fiber. Indeed, the propagation of the laser pulse through the fiber can be described by a linear phase transfer function. To determine the value of the fiber length which cancels out the FM-to-AM conversion induced by the propagation between the two gratings, amplified by the frequency conversion, we use the equation presented in [5]. With a $320 \mathrm{~m}$-long PMF, we obtain an excellent precompensation of AMs induced by the grating. Actually, the resulting second-harmonic pulse has no AMs at $f_{m}$ [cf. Fig. 2(1)].

To precompensate the shape of the amplitude filter due to the spectral acceptance of the crystal, we use the interferometric filter presented in the Fig. 1. This filter has three adjustable parameters: the fiber length, the angle $\delta$ and the parameter $\psi$. With the following parameters: $L_{\text {fiber }}=2 \mathrm{~m}, \delta=17^{\circ}$, and $\psi=\pi$, we obtain a good compensation of a large part of AMs induced by the KTP crystal at $2 f_{m}$. Indeed, the resulting distortion criterion of the second harmonic pulse is $\alpha_{F C S}<12 \%$ [cf. Figs. 2(d) and 2(h)] [5]. Note that it is theoretically possible to decrease this value with a more complicated interferometric filter.

In the temporal domain, the all-fiber system preinduces AMs at $1053 \mathrm{~nm}$, which are amplified by the crystal to compensate AMs due to the FCS [4]. We have studied by simulation the possibility of implementing this filter on the fibered part of the LMJ [6]. Simulations show that the amplitude of AMs at $1053 \mathrm{~nm}$ should be $<20 \%$ to obtain a good compensation at $351 \mathrm{~nm}$. This value is totally acceptable and should not enhance nonlinear processes at $1053 \mathrm{~nm}$ while reducing them at $351 \mathrm{~nm}$.

\section{CONCLUSION}

In conclusion, we have experimentally demonstrated the precompensation of FM-to-AM conversion due to a FCS with an all-fiber system. In the future, it would be interesting to study the experimental efficiency of such a precompensator system near the saturation of the frequency conversion process.

\section{References}

[1] J. E. Rothenberg, D. F. Browning, R. B. Wilcox, Proc. SPIE 3492, 51 (1999)

[2] S. Hocquet, D. Penninckx, E. Bordenave, C. Gouédard, Y. Jaouën, Appl. Opt. 47, 3338 (2008)

[3] R. W. Short, S. Skupsky, IEEE J. Quantum Electron. 26, 580 (1990)

[4] S. Vidal, J. Luce, D. Penninckx, Opt. Lett. 36, 88 (2011)

[5] S. Vidal, J. Luce, D. Penninckx, Opt. Lett. 36, 3494 (2011)

[6] S. Vidal, J. Luce, S. Hocquet, C. Gouédard, P. Calvet, D. Penninckx, Appl. Opt. 51, 5818 (2012) 